\title{
POSSIBLE CASES OF AFFINITY IN COTTON
}

\author{
MARGARET E. WALLACE \\ Department of Genetics, University of Cambridge
}

Received I 7.vii.59

\section{INTRODUCTION}

THE rapid re-establishment of plants of the parental species, after interspecific crossing, is a phenomenon very familiar to cotton-breeders and geneticists. There is abundant evidence of its occurrence in a number of situations: on inbreeding interspecies hybrids, on backcrossing their progeny to either parent species, and on growing in a mixed cultivation members of interfertile species. (Stephens, 1950, is a convenient source of references.)

On Harland's theory (1933 et seq.), each species evolves its own "architecture" of modifier systems and dominance mechanisms, and only those segregants survive, in generations following crossing, which receive complexes approaching either parental " architecture". This theory has been long accepted and found useful.

More recently, Stebbins (1945, 1947) has put forward the idea of "cryptic structural differentiation"; this envisages small sections of chromosomes rather than point mutations as the origin of differences between species. The idea is strongly supported by Stephens (1950), who has collected substantial cytological evidence, and some genetic evidence (1949). He takes the idea further than Stebbins, suggesting that, because of their structural differences, the paired chromosomes of species-hybrids are not completely homologous, so that crossing-over tends to produce deficient gametes and correspondingly defective or unbalanced zygotes.

While this process would favour the preservation of each chromosome as a whole, some workers doubt that it accounts for the rapid re-establishment of the parental types in the species under discussion, for cotton has a relatively high chromosome number; in the New World species there are twenty-six independent pairs which would still assort at random. Stephens' effect would be strengthened if there were also a mechanism tending to keep the chromosomes together.

Such a mechanism, in the form of the theory of " affinity", has in fact been proposed by Michie (r 953 et seq.) and Wallace (r 953 et seq.), to explain certain non-random associations of individual markers (or "quasi-linkage") in mice. It focuses on the centromere, since the function of this organelle appears to be to initiate the passage of the paired chromosomes to opposite poles at the first division of meiosis. It proposes that centromeres of the same ancestral origin tend to travel to the same pole; thus all the genes of the same origin tend to travel together-except those which are recombined with their centromeres. 
Quasi-linkage has been observed not only in the $F_{1}$ of crosses between distantly related individuals in mice but also in laboratory stocks which are many generations removed from the crosses by which their several mutants were combined. Here, there is no need to call upon the selection of modifier systems or the reduced survival of defective zygotes by crossing-over within structurally differing homologues. It is clear then that affinity can operate alone; on the other hand, if it operates in the process of re-establishment of parental species after hybridisation, its effect would be, and very probably is, enhanced by these phenomena.

The finding of affinity in species other than mice would be of considerable interest. It is most clearly discerned as the non-random segregation of markers known to be independent; but unfortunately it does resemble linkage. Certain discriminative features are therefore noted below. (These have been considered in detail elsewhere, Wallace, 1958.)

(i) Association of markers known to be independent : recombination values may be less than 50 per cent. or more, depending on centromeric phase. Recombination in excess of 50 per cent. (reversal) is particularly discriminative since only in the case of chromatid interference can this occur in chromosomal linkage and then it is not usually expected to exceed $5^{6}$ per cent. (Wright, 1947; Fisher et al., I947; Owen, I953).

(ii) Non-linear linkage and quasi-linkage relations of segregating markers.

It is thought on theoretical grounds that the greater centromeric differentiation should occur between rather than within species. Accordingly distinctive evidence for affinity would be the finding, on the one hand, of data exhibiting both of the features listed above in descendants of crosses between distantly related individuals (such as species-hybrids or the $F_{1}$ of inter-varietal crosses), and, on the other, of complete independence (rather than "loose linkage") of such quasi-linked markers in data from more closely related indiziduals (such as members of the same species or variety or selfed line).

Sir Joseph Hutchinson has drawn my attention to a possible case of quasi-linkage in the tomato and to several in cotton. The tomato data (Sawant, I958) is treated elsewhere (Wallace, I960a). The possibility of affinity in cotton would be of particular interest to the student of evolution, for the process of speciation has probably been more thoroughly examined in this genus than in any other organism. If present, affinity must play a part; whether it is largely causative or largely a result of the process would then have to be considered.

\section{THE DATA}

(i) The blackarm genes $B_{2}$ and $B_{3}$

(a) Knight's data and the linkage interpretation. $B_{2}$ and $B_{3}$, the mimic genes for resistance to blackarm disease, were transferred by Knight (I944) from G. punctatum (Bar 3) to G. barbadense (Sakel). After the 
initial outcross, repeated coupling backcrosses to barbadense were made, and $\mathrm{F}_{2}$ progenies were grown from several of the backcrosses. Special repulsion backcrosses were made up, also from various coupling backcross progenies. The progenies from the early backcrosses and the $\mathrm{F}_{2}$ progenies gave non-random assortment, and the later ones random or nearly random assortment; the repulsion data agreed according to its early or late origin. A similar programme, though less extensive, was carried out using another punctatum strain (Gambia Native), and similar results were obtained.

The data from the first four Bar 3 backcrosses were homogeneous and gave a recombination value of about 32 per cent., very significantly different from 50 per cent. ( $\chi_{1}^{2}$ testing independence exceeds 300 ). This led Knight to conclude that the genes are in fact linked. The "loss of linkage" on approaching the barbadense parent is also very marked: the last three backcrosses were not homogeneous and gave recombination values varying between $3^{2}$ per cent. and 48 per cent. $\chi_{1}^{2}$ testing their agreement with the earlier data is 18.27 ; thus there is no doubt that a change occurred at about the fifth backcross. Knight, then, logically attributes the loss to the replacement of the punctatum segment between $B_{2}$ and $B_{3}$ (hitherto maintained in each generation by the selection of coupling heterozygotes) by a barbadense one by double crossing-over. His assumption that crossing-over between homologues of different strains is inhibited has been amply supported in other contexts (Knight, I950, pp. 242, 243, gives several instances, of which two are described in detail by Stephens, I950, pp. I27, I 28).

Knight was aware of the possibility, in using mimic genes, of obtaining linkage-like effects by other processes than linkage-linkage is, in fact, discernible in all parts of the data as departures from the ratios expected, on independence, within only two classes: resistant and susceptible. At several of the backcross stages, he therefore made up putative single-intercross matings from the phenotypes most likely to be $B_{2}$ only and $B_{3}$ only, as discerned by their grade of resistance, and in each case he obtained clearly the single-factor ratio expected; thus the co-existence of the two genes at all stages was demonstrated. Moreover, the crosses are dated, showing that the "loss of linkage" occurred in different years in different stocks. Genetic misclassification and environmental factors are thus very unlikely to account for these striking results.

However, the reality of relatively loose linkage between mimic genes with a variable expression can never be fully accepted without evidence from other loci, preferably one located between the two. Unfortunately no linkages are known for either of these genes.

This being the case, the offer of an explanation of Knight's findings in terms other than misclassification or linkage, may seem to be of academic interest only. Nonetheless the possibility of affinity is discussed below because the situation described is, apart from the accident 
of mimicry, simple and of a kind peculiarly amenable to the demonstration of an affinity interpretation. It is hoped that, since transference of desirable genes from one strain or species to another has been the aim of many extensive programmes in cotton, geneticists other than the present writer may know of similar situations in published or unpublished data.

(b) An affinity interpretation. On the hypothesis that the linkage-like association between $B_{2}$ and $B_{3}$ is affinity rather than chromosomal linkage, $B_{2}$ and $B_{3}$ are situated near the centromeres of non-homologous punctatum chromosomes. The barbadense centromeres of these two chromosomes have a different centrotype, and the association between $B_{2}$ and $B_{3}$ in the early data is due to the tendency of the punctatum and barbadense centromeres to travel to opposite poles at meiosis $\mathrm{I}$. The genetic constitution of the hybrid $F_{1}$ and of its barbadense mate may be written respectively as

$$
\frac{B_{2} \alpha}{+\beta} \frac{B_{3} \alpha}{+\beta} \text { and } \frac{+\beta}{+\beta} \frac{+\beta}{+\beta}
$$

where $\alpha$ are punctatum and $\beta$ barbadense centromeres. The selection of $B_{2} B_{3}$ progeny in each generation to make the backcross or intercross (by selfing) of the next generation ensures that the heterocentricity of the heterozygote is maintained-for the gamete $B_{2} \alpha B_{3} \alpha$ results from simultaneous non-recombination between markers and centromeres and between centromeres. At some stage, however, crossingover will occur, and this in the $B_{2} B_{3}$ gamete must be, as in Knight's linkage hypothesis, double crossing-over, i.e.

$$
\begin{aligned}
& \text { (i) } \frac{B_{2} \beta}{+\beta} \frac{B_{3} \beta}{+\beta} \text { or (ii) } \frac{B_{2} \alpha}{+\beta} \frac{B_{3} \beta}{+\beta} \text { or (iii) } \frac{B_{2} \beta}{+\beta} \frac{B_{3} \alpha}{+\beta} \\
& B_{2} / \mathrm{C}_{2}, B_{3} / \mathrm{C}_{3} \quad B_{3} / \mathrm{C}_{3}, \mathrm{C}_{2} / \mathrm{C}_{3} \quad B_{2} / \mathrm{C}_{2}, \mathrm{C}_{2} / \mathrm{C}_{3},
\end{aligned}
$$

where $\mathrm{C}_{2}$ is the centromere linked to $B_{2}$ and $\mathrm{C}_{3}$ that linked to $B_{3}$. Since in all three cases at least one pair of homologues is homocentric, there will be no preferential segregation of centromeres at the first meiotic division and consequently independent assortment of $B_{2}$ and $B_{3}$ in the gametes. Double heterocentricity cannot be regained in later generations, since $\beta$ centromeres only are supplied by the barbadense mate; the "loss of linkage" is thus permanent.

If the centrotypes of the parents of the "repulsion" heterozygotes and thus of the heterozygotes themselves are deduced in a similar manner, the fit of expectation to observation in the repulsion data is found to be as adequate as in the coupling.

It is thus apparent that the data fit an affinity explanation as well as they do one of chromosomal linkage.*

* A certain difficulty must be mentioned, but it is one which militates against both hypotheses equally. At first sight it appears from the data that the change in association occurs abruptly at the fifth backcross of Bar $3 \times$ Sakel and that several double-crossovers, on either theory, must be invoked simultaneously; this seems unlikely. However, a 
Data which would discriminate between the two are of various kinds. For example, the linkage of a third factor to $B_{2}$ and $B_{3}$, allowing of a linear arrangement of the three genes on current interference concepts, would indicate linkage; whereas such a factor showing associations which do not allow of a linear arrangement would indicate affinity-and it might allow the location of the centromere in one pair of homologues. Again, by suitable breeding from the coupling backcrosses showing association of $B_{2}$ and $B_{3}$, repulsion heterozygotes could be made up some of which might give more than $5^{\circ}$ per cent. recombination, thus indicating affinity.

It is interesting that there are intraspecific data showing 50 per cent. recombination (Knight, I950, quotes "Cuany, unpublished"); this, in conjunction with the interspecific value of 32 per cent., would be taken in mice as evidence for affinity rather than of linkage. Its value in cotton, however, is open to some doubt in view of the known depression of crossing-over in interspecific data. Such depression is also found in the tomato (Sawant, I958). If widespread, it will give rise to the paradoxical situation that it can both help and hinder the case for affinity. For, on the one hand, reduction of crossing-over from marker to centromere increases the frequency with which the marker will share the centromere's preferential assortment with other centromeres and their markers-and so it extends the range of loci capable of showing quasi-linkage; while, on the other hand, for any one case which depends solely on the disparity between quasi-linkage from interspecific data and independence from intraspecific, it provides the alternative hypothesis of loose chromosomal linkage in both sets of data. In the latter instance, the case for affinity must rest on the proposition that the observed disparity is greater than may be expected on experience of chromosomal linkage.

The disparity may be expressed as $\frac{x^{\prime}}{x}$, where $x^{\prime}$ and $x$ are the mapdistances corresponding to the intraspecific recombination value, $y^{\prime}$, and the interspecific value, $y$, respectively. This measure is reasonable since the map-distance in a given physical segment in the interspecific heterozygotes is proportional to the number of cross-overs observed, and in the intraspecific ones, where this number is less, the proportionality presumably still applies. The transformations $y^{\prime}$ to $x^{\prime}$ and $y$ to $x$ must be based on a degree of interference suitable to the case under study. In the present one, the Kosambic conversion $x=\frac{1}{2} \tanh ^{-1}(2 y)$ is used in the absence of observations on interference because it has been found to have wide application. Choosing

thorough analysis by the writer reveals that this difficulty is not present in the $F_{2}$ and repulsion parts of these data nor in the second series of crosses (Crambia Native $\times$ Sakel), and that, on certain plausible assumptions, a satisfactory explanation may be found where it does occur. 
(from Knight, 1950) the chromosomal linkage cases in which there is the greatest disparity and thus this fraction is the largest we have:

\begin{tabular}{|c|c|c|c|c|}
\hline & & \multicolumn{2}{|c|}{ Known chromosomal linkages } & \multirow{2}{*}{$\begin{array}{c}\text { Possible affinity } \\
B_{2}-B_{3}\end{array}$} \\
\hline & & $L-L c_{1}$ & $R_{1}-c l$ & \\
\hline $\begin{array}{l}\text { Recombination : } \\
\quad \text { Intraspecific, } y^{\prime} \\
\text { Interspecific, } y \\
\text { Measure of disparity, } \frac{\dot{x^{\prime}}}{x}\end{array}$ & $\begin{array}{l}\cdot \\
\dot{ } \\
\cdot\end{array}$ & $\begin{array}{l}29 \cdot 9 \text { per cent. } \\
24.5 \text { per cent. } \\
\quad \text { I } 25\end{array}$ & $\begin{array}{l}\text { I6 per cent. } \\
8 \text { per cent. } \\
2 \cdot 0\end{array}$ & $\begin{array}{l}45-50 \text { per cent. } \\
32 \text { per cent. } \\
2 \cdot 0 \text { to } \infty\end{array}$ \\
\hline
\end{tabular}

The lowest linkage value tolerable by the independence data for $B_{2}$ and $B_{3}$ is not given, but 45 per cent. is chosen as a possibility in order to give the smallest disparity. In other words, in presenting the information above, the case for affinity has been made as weak as possible. Despite this, in the $B_{2}-B_{3}$ case the disparity is as large as, or larger than, in the others, and so affinity survives as the more plausible hypothesis.*

(c) Conclusions. Accepting affinity, it is worth considering what Knight's results imply both for the cottons used and more generally. (i) For those centromeres linked to $B_{2}$ and $B_{3}$-and for others also, if cumulative attraction is operating (see section 2a)-the punctatum variety of hirsutum differs from the barbadense. (These correspond to the $(\mathrm{AD})_{1}$ and $(\mathrm{AD})_{2}$ genomes respectively, but it cannot be deduced without further evidence, whether these statements can be applied to the whole genomes also.) (ii) In general, loose linkages based on interspecific data only, cannot be accepted as unquestionably chromosomal, particularly if the data are from heterozygotes of only one phase.

\section{(ii) The L-Lc $c_{1}$ linkage group}

(a) Hutchinson's data. This group was established by Hutchinson (1934) while investigating the relation of the leaf-shape alleles ( $L$ locus). Lint-colour ( $L c_{1}$ locus) $\dagger$ was found to be linked with $L$ with about 30 per cent. recombination (Ibid. p. 491). These data, which involve as many as eight strains of $G$. arboreum, are homogeneous; this situation alone makes an affinity explanation very unlikely and there has since been confirmation of linkage by the location of the marker $l i_{d}$ between the two (Govande, I944).

By contrast, data involving $L$ and the anthocyanin locus $R_{2}$, also from crosses within arboreum, while formally homogeneous, are

* It is impossible to devise a satisfactory test of the excess of the disparity for one case over that of another. This is because, when the intraspecific $y^{\prime}$ value expected in the case of affinity is most nearly realised, i.e. at or near 50 per cent., $x^{\prime}$ tends to infinity.

$\uparrow$ The symbols suggested by Knight (1950, Appendix II) are used throughout this paper. 
found to give rather different recombination values. Three backcrosses give, singly and together, an insignificant deviation from 50 per cent., while the fourth gives a very significant one $\left(\chi^{2}{ }_{1}=10 \cdot 0\right)$, the recombination value being 40 per cent. The heterozygotes in the first three backcrosses came from crosses within and between the closely related varieties bengalense and burmanicum, while the heterozygote in the fourth backcross came from a cross between the more distantly related varieties bengalense and soudanense (strain G.S.2 of bengalense and Abu Hareira of soudanense).

This suggests that $L$ and $R_{2}$ are situated near different centromeres, the heterozygotes in the first three backcrosses having only one kind of centromere and thus showing independence, and that in the fourth having more than one, i.e. being a convergent heterocentric and thus showing the quasi-linkage. Thus at least two centromeres differ between the two strains used in the fourth cross.

So far the observations may be viewed as a typical case of affinity. However, there are further data in this paper, which, though less significant, are worth considering in view of Silow's work (section $b$ ), and these do not admit of so clear and single an interpretation. One of the heterozygotes in the three backcrosses described above, and a selfed heterozygote from the same source, were segregating in $L c_{1}$ also. While the value for $L-R_{2}$ is insignificantly less than $5^{\circ}$ per cent. ( 45.9 per cent.), that for $R_{2}-L c_{1}$ significantly exceeds it ( 56 per cent., $\left.\chi^{2}>6\right)$. This may be represented diagrammatically:

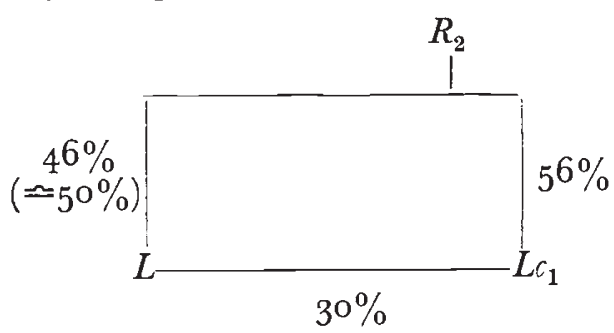

The relations $R_{2}-L c_{1}$ suggest that affinity is operating here too, this quasi-linkage being a reversal. $R_{2}$ and $L c_{1}$ must then be near their centromeres, and these in the heterozygote are divergent. As $L$ is linked to $L c_{1}$ it should also show reversal with $R_{2}$. The data do admit of a value $L-R_{2}$ more than 5 o per cent., but only slightly more, which indicates that the centromere of the $L-L c_{1}$ chromosome is nearer $L c_{1}$ than $L$. The situation may be represented as follows:

$$
\begin{aligned}
& \text { heterozygote } \\
& \frac{L L c_{1} \alpha}{++\beta} \frac{R_{2} \beta}{+\alpha}
\end{aligned}
$$

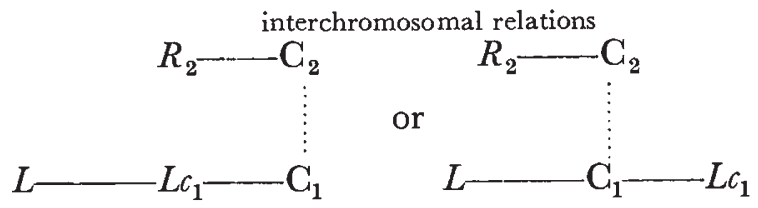

where $\mathrm{C}_{1}$ is the centromere near $L c_{1}$ and $\mathrm{C}_{2}$ that near $R_{2}$, and each can be of the two centrotypes $\alpha$ and $\beta$. (The $\alpha, \beta$ designation is not, of 
course, intended to imply any equivalence of centrotype with the centromeres supposedly linked to $B_{2}$ and $B_{3}$ in Knight's data.)

Before this interpretation can be accepted, two questions must be answered: (i) is the $L-R_{2}$ value in the bengalense $\times$ soudanense heterozygote, about 40 per cent., compatible with the one here which is much nearer $5^{\circ}$ per cent.? (ii) is the supposition of two centromeres each of two centrotypes compatible with the origin of the heterozygote, mainly * from within burmanicum, which is so far assumed to be of only one centro-type?

On question (i): the same deviation from independence cannot, strictly, be expected unless the crosses are identical. In the present case, the bengalense $\times$ soudanense cross is known to be the wider and it is reasonable to expect the deviation from 50 per cent. to be correspondingly wider. Either or both of two factors may be responsible: depression in crossing-over between the markers and their centromeres, or increased attraction between the similar centromeres. Increased attraction may result from a greater difference between homologous centromeres of widely separated varieties, or from the possibility that the centromeres of more chromosomes are different and that they act cumulatively (Wallace, I958, p. 219. While depression of crossingover in cotton hybrids is well known, the idea of increased centromeric attraction has not been tested experimentally).

On question (ii): the assumption that burmanicum has only one kind of centromere is not strictly necessary. To explain the soudanense $\times$ bengalense result it must be postulated that those in bengalense are different from those in soudanense but this explanation is not weakened by the supposition of differences also though smaller or involving fewer centromeres) within burmanicum since the strains concerned (Burma Ghost and Burma Laciniated) are morphologically very different. This supposition may, however, seem to be stretching the theory solely to accommodate a not outstandingly significant reversal. Indeed the reversal demands not only that the ancestral sources of the two chromosomes concerned have different centromeres but that there has been an interchange of these centromeres at some stage before the cross producing the divergent heterozygote. However, on hearing that the data require the postulation of an interchange, Sir Joseph has revealed that the two plants contributing the relevant chromosomes of the heterozygotes were derived from a bulked plot of several strains of burmanicum gathered from a wide range of localities in Burma, and that these plants were labelled Burma Ghost and Burma Laciniated solely on their appearance: they may well have arisen after crossing between some of these strains. Thus the means of interchange, namely crossing, is shown to have been very likely.

It must be pointed out that it is still not possible to discern, without experiment, whether or not burmanicum as a whole contains more than

* The pedigree given the writer shows that some contribution ultimately came from a bengalense plant but that it is very unlikely to have involved the chromosomes concerned here. 
one kind of centromere; for it might be that interchange with other varieties has introduced new centromeres to it, rather than that the two strains of burmanicum concerned here have evolved differing centromeres: natural crossing between varieties whose habitats overlap, such as burmanicum and bengalense, cannot be excluded. The important point here is that quasi-linkage with an excess of recombinants should not be unexpected, and that in general, barring recent special crossing by breeders and geneticists, it may be expected in hybrids of strains, varieties or species which can cross naturally or have been able to do so in the not too remote past, i.e. since their divergence. The tomato data already mentioned exhibit a reversal, and here, while it is not possible to establish when the interchange occurred, the postulation of an interchange does provide an adequate explanation of several morphological and physiological relations between the varieties involved.

(b) Silow's data. Silow's data (I94I) concern outcrosses of the cultivated cottons $G$. herbaceum and $G$. arboreum to the wild $G$. anomalum, and backcrosses to various strains of the cultivated cottons (not always the same species or strains in any one series of backcrosses). He established clear differences between the species at several loci, most of his data being mono-factorial. Incidental bi-factorial data, and some obtained specifically to discern linkage, are reported (Ibid. p. 315). All the data concern the segregation of alleles at the anthocyanin locus $R_{3}$, with those at eight other loci.

Most of the data are from late backcross generations, that is, rather too remote from the initial outcross to expect that any heterocentricity present initially can have been maintained (see section I). Some of the data are from heterozygotes whose ancestry the writer was not able to discern very certainly from the text, but it seems likely that they were late backcrosses. Of the fifteen sets of backcross data given, most do in fact show independence, and the total ratio of non-recombinants to recombinants (pooling for all loci) shows close agreement with independence.

However, one segregation, while not deviant enough to make the total data heterogeneous, does catch the eye as showing an individually very significant departure from independence. It concerns $R_{3}$ and $L c_{1}: \chi_{1}^{2}{ }_{1}$ is $7 \cdot 2$ and the recombination value 62 per cent. Inspection of the origin of the heterozygote shows that of the fifteen, this one's ancestry is the most mixed, anomalum, arboreum and herbaceum having all been involved. This heterozygote (the only repulsion one) may be represented as divergent in the usual way:

$$
\frac{R_{3} \alpha}{+\beta} \frac{+\beta}{L c_{1} \alpha}
$$

(Again, no identity of a centromere labelled $\alpha$ here with one similarly labelled elsewhere, is intended: the $\alpha$ centromeres are merely those which tend to travel to one pole, the $\beta$ ones those that travel to the 
other. Since three species have been involved in the present data, there may be three types of centromere, but there is no way of knowing from the text which are linked with each allele, and thus which show preferential tendencies.)

$R_{3}$ is not the same locus as $R_{2}$ of Hutchinson's data and they are probably in different homologues; but $L c_{1}$ is again concerned, and again showing recombination exceeding 50 per cent. This being so, the segregation of $L$ and $R_{3}$ is of interest: it gives independence. This could be taken as confirmation that $L$ is farther from the centromere than $L c_{1}$, but for the fact that it occurs in a different heterozygote where independence is probably due to partial homocentricity. One may merely observe that $L c_{1}$ is concerned in a quasi-linkage in Silow's data as it is in Hutchinson's, but here more significantly. This is, then, confirmatory evidence for affinity.

However, as is to be expected from experiments not designed specifically to test it, affinity is not the only interpretation for both bodies of data. The observed relations between $L-L c_{1}$ and $R_{2}$ and between $L-L c_{1}$ and $R_{3}$, are rather similar and may be summarised diagrammatically:
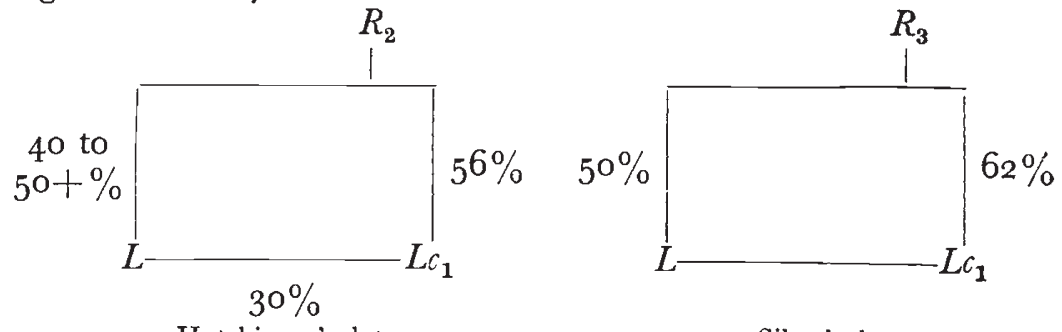

Hutchinson's data

Silow's data

On the assumption of chromatid interference, $R_{2}-L c_{1}$ and $R_{3}-L c_{1}$ could be chromosomal linkages. The $L-L c_{1}$ group then becomes $R_{2}-L-L c_{1}$ and $R_{3}-L-L c_{1}$. However, both of these situations cannot be true since $R_{2}$ and $R_{3}$ are not allelic nor closely linked, so that affinity may be accepted for at least one of them. A discriminating experiment for the $R_{2}-L-L c_{1}$ trio is being planned by $\mathrm{Mr} \mathrm{J} . \mathrm{H}$. Saunders at Shambat, Khartoum.

It is hoped that those more conversant with cotton genetics than the writer may know of other cases, published or otherwise, which fit an affinity interpretation. In the present case, independence data, or data showing less than 50 per cent. recombination, for the $L-L c_{1}$ group and either $R_{2}$ or $R_{3}$, would, according to its origin, throw light on the real nature of their relationship.

Since an experiment will usually be necessary in order to test suggestive cases of affinity, it may be useful here to outline two kinds which can probably be carried out in cotton without much preparation. (This subject has been considered in some detail elsewhere, Wallace, I958.) Both involve the outcross of widely separated strains, varieties or species, to produce hybrids heterozygous for several factors, and both 
require that these be backcrossed to a multiple recessive stock (which need have no special relation with the parental stocks).

In one kind - which is suitable for the $R_{2}-L-L c_{1}$ situation - the hybrid contains at least one pair of linked factors and one or more factors not linked with these: they are chosen, if possible, on evidence such as has been described here, as being centromere markers. If their joint segregation gives linkage and quasi-linkage relations which do not allow of a linear relation between the markers, not only is an affinity hypothesis indicated but the position of the centromere relative to the two linked markers may be determined-or, if more than one pair of linked markers shows quasi-linkage, the position of the centromere relative to each pair may be determined.

In the second kind, the hybrids are heterozygous for as many independent markers as possible. If their joint segregation gives quasi-linkages, these not only identify centromere markers but supply information on centromeric differences, and possibly upon interchanges, between the strains or species used. If no quasi-linkages are obtained from interspecific data, then either (i) the markers used are not sufficiently near their centromeres, or (ii) the centromeres of the two strains or species used are the same, or (iii) the case for affinity in cotton is weakened. All these possibilities are of obvious interest. The results of this kind of experiment in mice, using differing inbred strains, are given elsewhere (Wallace, I $960 b$ ).

(c) Conclusions. If the relation between $L$ and $R_{2}$ in Hutchinson's data is accepted as affinity, then

(i) these loci are near their centromeres and there are centromere differences within arboreum, viz., between the varieties bengalense and soudanense.

If the (less significant) relation between $L c_{1}$ and $R_{2}$ in the same data is also accepted as affinity, then

(ii) $L c_{1}$ is probably nearer its centromere than is $L$, and there may be centromeric differences within burmanicum.

Accepting affinity in Silow's data,

(iii) $R_{3}$ is near its centromere and there are centromeric differences between anomalum and either or both of herbaceum and arboreum.

These conclusions also imply

(iv) linkage group I $\left(L-L c_{1}\right)$ is in a different chromosome from groups $7\left(R_{2}\right)$ and $6\left(R_{3}\right)$.

Acknowledgment.- It is a pleasure to record my debt to Sir Joseph Hutchinson for drawing my attention to the existence of cotton data capable of an affinity interpretation, and for several fruitful discussions.

\section{REFERENCES}

FISHER, R. A., LYON, M. F., AND OWEN, A. R. G. I947. The sex chromosome in the house mouse. Heredity, $I, 355-365$.

GovANDE, G. K. I 944. Linkage relations of the $l i_{d}$ gene for lintlessness in the Asiatic cottons. Curr. Sci., I3, $32 \mathrm{I}$. 
harland, s. c. I933. The genetical conception of the species. Mem. Acad. Sci. U.S.S.R. No. 4 (reprinted in Trop. Agr., II, 5I-53).

Hutchinson, J. в. I934. The genetics of cotton. X. The inheritance of leaf shape in Asiatic Gossypiums. 7. Genet., 28, 438-51 3 .

KNIGHT, R. L. I944. The genetics of blackarm resistance. IV. Gossypium punctatum crosses. F. Genet., 46 , I-26.

KNIGHT, R. L. I950. Abstract bibliography of cotton breeding and genetics, I900I950. Tech. Comm. I 7 of the Commonwealth Bureau of Plant Breeding and Genetics, Cambridge.

michie, D. I953. Affinity: a new genetic phenomenon in the house mouse. Nature, ${ }_{171}, 26-27$.

oWEN, A. R. G. 1953. Super-recombination in the sex chromosome of the mouse. Heredity, 7, I03-I Io.

SAWANT, A. C. I958. Cytogenetics of interspecific hybrids, Lycopersicon esculentum Mill. and L. hirsutum Humb. and Bonpl. Genetics, 43, 502-515.

silow, R. A. I94I. The comparative genetics of Gossypium anomalum and the Asiatic cottons. F. Genet., 42, 259-358.

Stebbins, G. L. I945. The cytological analysis of species hybrids. II. Bot. Rev., $I I, 463-486$.

STEBBiNs, G. L. I947. Types of polyploids : their classification and significance. In Demerec, M., Advances in Genetics, I, 403-429.

Stephens, s. G. 1949. The cytogenetics of speciation in Gossypium. I. Selective elimination of the donor parent genotype in interspecific backcrosses. Genetics, $34,627-637$.

STEPHENS, s. G. 1950. The internal mechanism of speciation in Gossypium. Bot. Review, 16 , I I5-149.

WALlACE, M. E. I953. Affinity : a new genetic phenomenon in the house mouse. Nature, $171,27-28$.

WALlACE, M. E. I958. Experimental evidence for a new genetic phenomenon. Phil. Trans. Roy. Soc. B, 24I, 2 I I-254.

WALlACE, M. E. I I 60a. A possible case of affinity in tomatoes. Heredity, I4, 275-283. WALLACE, M. E. I96o $b$. Affinity : evidence from an outcrossing programme in mice. I. Results from the use of segregating inbred lines. (In preparation.)

WRIGHT, M. E. (= WALLACE). I947. Two sex-linkages in the house mouse with unusual recombination values. Heredity, $I, 349-354$. 